\title{
Biological properties of bone marrow-derived early and late endothelial progenitor cells in different culture media
}

\author{
XIU M. GUAN ${ }^{*}$, MIN CHENG ${ }^{*}$, HONG LI, XIAO D. CUI, XIN LI, YU L. WANG, \\ JIN L. SUN and XIAO Y.ZHANG
}

\author{
Medicine Research Center, Weifang Medical University, Weifang, Shandong 261053, P.R. China
}

Received March 27, 2013; Accepted September 26, 2013

DOI: $10.3892 / \mathrm{mmr} .2013 .1718$

\begin{abstract}
Ex vivo expansion of endothelial progenitor cells (EPCs) may be a promising strategy to overcome the clinical problem of limited cell numbers. As the culture medium is the key for the cell characteristics, the effects of different culture media on EPCs were investigated in the present study. Rat bone marrow mononuclear cells were cultured in different media, including M-199 media with 20\% fetal bovine serum (FBS) and bovine pituitary extract (M1); M-199 media with $10 \% \mathrm{FBS}, 20 \mathrm{ng} / \mathrm{ml}$ vascular endothelial growth factor (VEGF) and $10 \mathrm{ng} / \mathrm{ml}$ basic fibroblast growth factor (bFGF; M2) or epidermal growth medium (EGM)-2MV media. The cell morphology and biological functions, such as proliferation, adhesion, migration, tube formation and nitric oxide (NO) production were subsequently assayed in vitro. Moreover, endothelial biomarkers and apoptosis were also analyzed. The results showed that endothelial-like cells appeared in all of the culture systems. First-passage cells, namely early EPCs, tended to form colonies in M2 and EGM-2MV media but showed a fusiform shape in M1 media. The 3rd or 4th generation EPCs, namely late EPCs, cultured in EGM-2MV media exhibited increased adhesion, migration, tube formation and NO production as compared with EPCs in M1 or M2 media. Furthermore, late EPCs cultured in EGM-2MV expressed higher levels of endothelial cell markers, such as von Willibrand factor (vWF) and CD31, but relatively greater levels of apoptosis were observed. In conclusion, cell culture conditions, for example the medium used, affects the biological properties of bone marrow-derived early and late EPCs.
\end{abstract}

Correspondence to: Dr Min Cheng or Dr Xiao Y. Zhang, Medicine Research Center, Weifang Medical University, Baotong Street No. 7166, Weifang, Shandong 261053, P.R. China

E-mail: chengmin1976@wfmc.edu.cn

E-mail: xiaoyun.zhang@126.com

*Contributed equally

Key words: endothelial progenitor cells, cell culture, mononuclear cells, bone marrow

\section{Introduction}

Loss of endothelial integrity and impaired capacity for neovascularization are hypothesized to contribute to cardiovascular diseases, such as atherosclerosis and ischemic events in the limbs, retina and myocardium (1). Recent studies have shown that endogenous re-endothelialization and postnatal neovascularization rely on the migration, proliferation and sprouting of preexisting mature endothelial cells, as well as on the activity of endothelial progenitor cells (EPCs) $(2,3)$. EPCs promote re-endothelialization or stimulate angiogenesis directly by differentation and proliferation, and also indirectly with secretory factors that mobilize endothelial and progenitor cells to be involved in angiogenesis and reconstruction $(4,5)$. Therefore, EPCs may be used as a potential therapeutic strategy in vascular disorders.

It has been recognized that EPCs are a heterogeneous population and, according to their morphology, function and growth potential, are classified into at least two different populations in ex vivo culture systems: Pro-angiogenic cells, namely early EPCs, and endothelial colony forming cells (ECFCs), also termed late EPCs. Early EPCs appear within 4 to 7 days of culture, are spindle-shaped and have a limited proliferation potential. Late EPCs develop after two to three weeks of culture and have a cobblestone appearance (6). Previous data suggest that late EPCs are closer to mature endothelial cells in phenotype but show notable proliferative, migrational and tube-forming capabilities; while early EPCs may contribute to endothelialization and neovascularization by the secretion of vasoactive substances, such as cytokines, stromal cell-derived factor-1 (SDF-1), nitric oxide (NO) and prostaglandin $\mathrm{I}_{2}$ (6-8).

Although the treatment of cardiovascular patients with EPCs may be a potential therapeutic option, the numbers of cells that are directly obtained from bone marrow, peripheral blood and umbilical cord blood are not large enough for use in a clinical setting. For example, the animal study results from Iwaguro et al (9) indicated that up to $12 \mathrm{~L}$ of autologous blood may be required to harvest sufficient EPCs in order to induce angiogenesis in patients following intravenous cell infusion. Thus, ex vivo culture and expansion of EPCs may be a promising strategy to overcome the clinical problem of limited cell numbers. However, EPCs which were cultured in different culture conditions exhibited different morphologies, surface markers and biologic functions. It is known that the culture 
medium is the key in the determination of cell characteristics. At present, the common media used for ex vivo expansion of EPCs, include M-199 with 20\% fetal bovine serum (FBS) and $0.05 \mathrm{mg} / \mathrm{ml}$ bovine pituitary extract $(10,11)$; M-199 with $10 \%$ FBS, $20 \mathrm{ng} / \mathrm{ml}$ vascular endothelial growth factor (VEGF) and $10 \mathrm{ng} / \mathrm{ml}$ basic fibroblast growth factor (bFGF) $(12,13)$; and endothelium growth medium (EGM)-2 MV medium (supplemented with EGM-2 bullet kit, including 5\% fetal calf serum, vascular endothelial growth factor (VEGF), R3-insulin-like growth factor 1 (R3-IGF-1), human recombinant epidermal growth factor (rhEGF), human recombinant fibroblast growth factor (rhFGF)-B, Gentamicin, Amphotericin-B (GA-1000), hydrocortisone and ascorbic acid) $(14,15)$. Therefore, the purpose of the present study was to investigate and compare the effects of these different culture media on the early and late EPC morphology and cell functions (proliferation, adhesion, migration, differentiation, secretion and tube formation).

\section{Materials and methods}

Isolation of bone marrow mononuclear cells and cell culture. Whole bone marrow was isolated from the femurs and tibias of Sprague-Dawley rats (weight, 150-175 g), which were obtained from Weifang Medical University, Shandong, China. The bone marrow mononuclear cells (MNCs) were fractionated by density gradient centrifugation (Histopaque ${ }^{\circledR}-1083$, Sigma-Aldrich, St. Louis, MO, USA). MNCs were plated on dishes precoated with fibronectin (Roche, Mannheim, Germany) and were cultured in M1 [M-199 (HyClone, Logan, UT, USA) with 20\% FBS (HyClone) and bovine pituitary extract (Sigma Aldrich Chemie, Schnelldorf, Germany)], M2 [M-199 with 10\% FBS, 20 ng/ml VEGF (PeproTech, Rocky Hill, NJ, USA) and $10 \mathrm{ng} / \mathrm{ml}$ bFGF (Sigma Aldrich Chemie)] or EGM-2MV (EGM-2 with EGM-2 bullet kit, including, $5 \%$ fetal calf serum, VEGF, R3-IGF-1, rhEGF, rhFGF-B, GA-1000, hydrocortisone and ascorbic acid) media. After four days in culture, unattached cells were removed by a single washing step with phosphate-buffered saline (PBS), following which, fresh medium was added. This study was conducted in accordance with the recommendations of the Guide for the Care and Use of Laboratory Animals of the National Institutes of Health. All animal protocols were approved by the local ethics committee at Weifang Medical University (Weifang, China; permit no. 5876).

Colony-forming assay. Isolated MNCs were resuspended in different growth media, and in total, $5 \times 10^{6} \mathrm{MNCs}$ were preplated in fibronectin-coated 6-well plates in duplicate. After two days, the non-adherent cells were collected and $1 \times 10^{6}$ cells were replated onto a fibronectin-coated 24-well plate. On the fifth day, the number of colony-forming units (CFUs)/well was counted for each sample. A colony of EPCs was defined as a central core of round cells with elongated sprouting cells at the periphery. All colonies were counted manually in a minimum of three wells by two independent investigators under blind conditions as described previously (16).

Cell proliferation assay. Cell proliferative activities were analyzed using cell counting kit-8 (CCK-8; Dojindo Laboratories, Kumamoto, Japan). Briefly, EPCs were seeded onto 96-well plates (density, 1,000 cells/100 $\mu \mathrm{l} /$ well), CCK-8 was added to each well according to the manufacturer's instructions and incubated for $1 \mathrm{~h}$ at $37^{\circ} \mathrm{C}$. The optical density (OD) value at $450 \mathrm{~nm}$ was measured using an enzyme-linked immunoabsorbent assay reader (Bio-Rad 680; Bio-Rad Laboratories, Hercules, CA, USA).

Cell differentiation assay. Total cellular RNA was isolated with TRIzol reagent (Invitrogen Life Technologies, Carlsbad, CA, USA) and reverse-transcribed to cDNA using the SYBR ${ }^{\circledR}$ PrimeScript ${ }^{\circledR}$ RT-PCR kit (Takara Bio Inc., Shiga, Japan) at $37^{\circ} \mathrm{C}$ for $15 \mathrm{~min}$. Endothelial cell differentiation markers, such as von Willibrand factor (vWF) and CD31, and gene expression were analyzed by SYBR ${ }^{\circledR}$ Premix Ex Taq ${ }^{\mathrm{TM}}$ (Takara Bio, Inc.). Primers used for amplification were as follows: Sense: 5'-GCG TGG CAG TGG TAG AGT A-3' and antisense: 5'-GGA GAT AGC GGG TGA AAT A-3' for vWF; sense: 5'-GAC AGC CAA GGC AGA TGC AC-3' and antisense: 5'-ATT GGA TGG CTT GGC CTG AA-3' for CD31; and sense: 5'-GGC ACA GTC AAG GCT GAG AAT-3' and antisense: 5'-ATG GTG GTG AAG ACG CCA GTA-3' for glyceraldehyde 3-phosphate dehydrogenase, which was used as a housekeeping gene, in order to normalize the expression target gene. The thermal cycling conditions were as follows: $30 \mathrm{sec}$ at $95^{\circ} \mathrm{C}$ for pre-denaturation, 40 cycles for $15 \mathrm{sec}$ at $95^{\circ} \mathrm{C}$ for denaturation, $1 \mathrm{~min}$ at $59^{\circ} \mathrm{C}$ for annealing and $10 \mathrm{sec}$ at $72^{\circ} \mathrm{C}$ for elongation. At the end of each cycle, the fluorescence emitted by SYBR-Green I was measured. Following the completion of the cycling process, samples were immediately subjected to a temperature ramp for melting curve analysis.

The protein expression of CD31 and vWF was also determined by a fluorescence-activated cell sorter (FACS, Becton Dickinson, Franklin Lakes, NJ, USA). Cells were trypsinized and incubated with CD31 or vWF antibody (eBioscience, San Diego, CA ,USA) for $1 \mathrm{~h}$. For the detection of vWF, the cells were permeabilized with $0.1 \%$ Triton X-100 prior to incubation with the antibody. Typically, 20,000 late EPCs were measured for fluorescence intensity per experiment. In addition, isotype controls were performed for each sample condition and the mean fluorescence intensity identified for the isotype control was subtracted from the mean fluorescent intensity of the antibody-bound cells.

Cell apoptosis assay. Approximately $1 \times 10^{6}$ cells were double-stained with Annexin V-fluorescein isothiocyanate and propidium iodide (PI) using an Annexin V-FITC Apoptosis Detection kit (Becton Dickinson) according to the manufacturer's instructions. Apoptotic cells (Annexin $\mathrm{V}^{+} / \mathrm{PI}^{-}$) were detected by FACS. The apoptotic percentage analysis was performed using Cell-Quest software (Becton Dickinson).

Cell adhesion assay. Cells were washed with PBS, and gently detached with $0.25 \%$ trypsin/EDTA. Following centrifugation and resuspension with serum-free medium, equal cell numbers were seeded on $50 \mu \mathrm{g} / \mathrm{ml}$ fibronectin-coated culture dishes, and incubated for $1 \mathrm{~h}$ at $37^{\circ} \mathrm{C}$. Cultures were washed three times with PBS to remove non-adherent cells. Then adherent cells were counted independently in six random high power (x100) microscope fields/well by three blinded observers. 

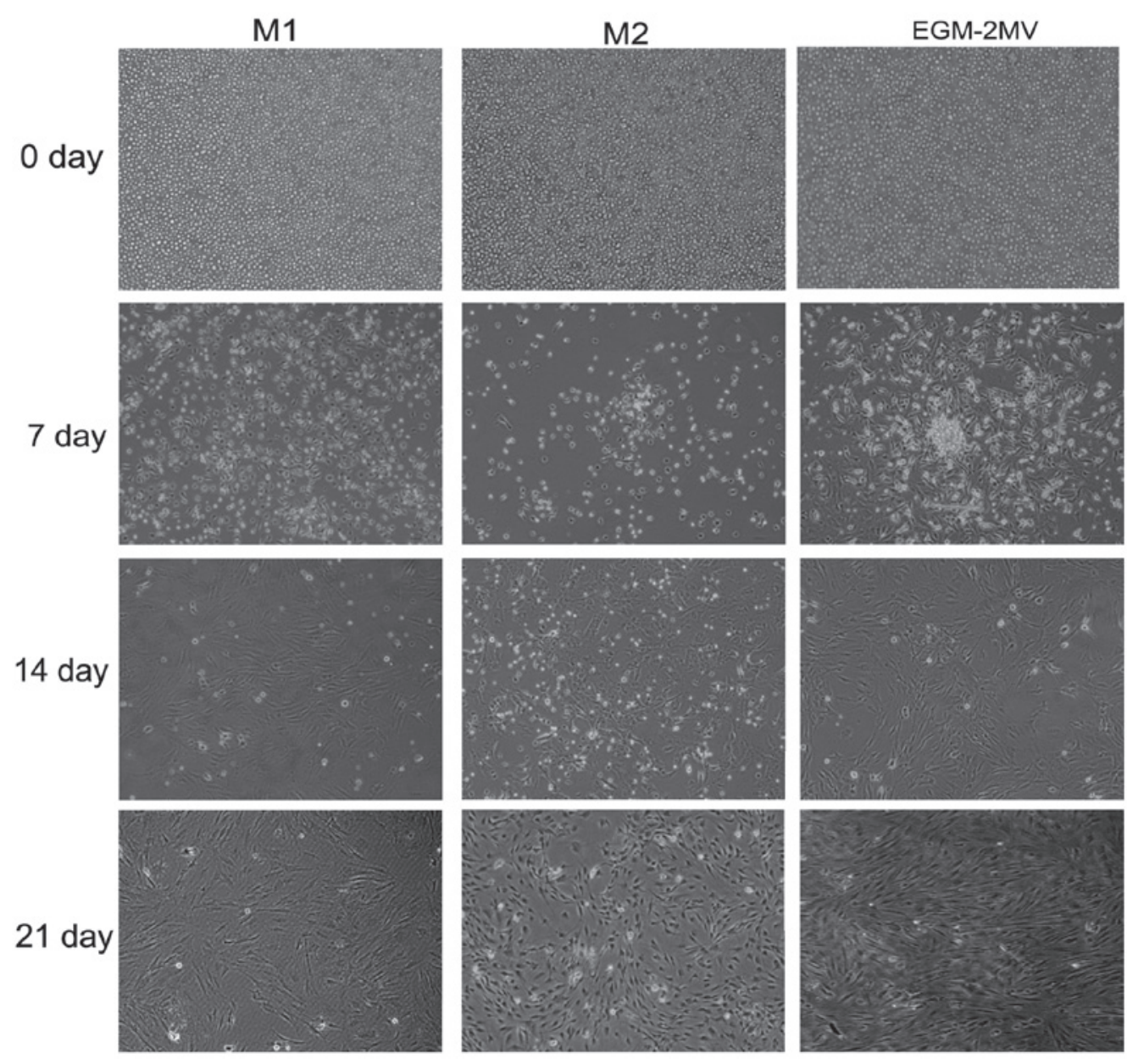

Figure 1. Morphological characteristics of endothelial progenitor cells (EPCs) in different culture media. Mononuclear cells (MNCs) isolated from rat bone marrow were plated on dishes precoated with fibronectin and cultured in M1, M2 or EGM-2MV media, respectively. MNCs initially cultured in the different media were round. Seven days following plating, the primary culture of MNCs started to differentiate into early EPCs and tended to form colonies with the round cells at the center and the typical spindle cells at the periphery. After 21 days, the third-forth passage cells (late EPCs) exhibited a cobblestone-like morphology (magnification, x100).

Cell migration assay. The migratory function of EPCs was analyzed by a modified Boyden chamber (CoStar, Cambridge, MA, USA) assay. Briefly, a total of $1 \times 10^{5}$ EPCs were placed in the upper chamber and medium containing with SDF-1 was placed in the lower chamber. The assays were conducted over a $16 \mathrm{~h}$ incubation period at $37^{\circ} \mathrm{C}$ in an incubator equilibrated to $5 \% \mathrm{CO}_{2}$. The membrane was then washed gently with PBS and fixed with $4 \%$ paraformaldeyde. Non-migrating cells were gently removed with cotton balls from the upper side of the membrane, and the membrane was then stained by using DAPI. The migration of late EPCs was analyzed by counting the number of migrated cells in six random high-power (x100) microscope fields/well.

In vitro tube formation assay. A 96-well plate was coated with $100 \mu 1$ Matrigel (Becton Dickinson) and incubated at $37^{\circ} \mathrm{C}$ for $1 \mathrm{~h}$. Late EPCs/ml $\left(2 \times 10^{5}\right)$ were added to each well for $10 \mathrm{~h}$. The enclosed networks of tubes were photographed from six randomly chosen fields under a microscope. The averages of the total number and area of complete tubes, formed by late EPCs, per unit area were compared by Image-Pro Plus (Media Cybernetics, Rockville, MD, USA).
NO concentration assay. The NO concentration in EPC culture supernatants was detected using an NO nitrate reductase assay kit (Nanjing Jiancheng Bioengineering Institute, Nanjing, China), according to the manufacturer's instructions. The NO concentration was calculated according to the following formula: NO $(\mu \mathrm{mol} / 1)=$ standard concentration (100 $\mu \mathrm{mol} / 1$ dilution factory of sample x (OD of NO measuring tube-OD of blank tube)/(OD of standard-OD of blank).

Statistical analysis. Unless otherwise indicated, results are presented as the mean \pm SE from at least three independent experiments. Statistical analyses were performed by one-way analysis of variance, followed by Tukey-Kramer post hoc test for multiple comparisons. $\mathrm{P}<0.05$ was considered to indicate a statistically significant difference. All data were analyzed using SPSS software (version 15.0; SPSS Inc., Chicago, IL, USA).

\section{Results}

Morphological characteristics of EPCs in different culture media. The bone marrow MNCs initially cultured in the 
A

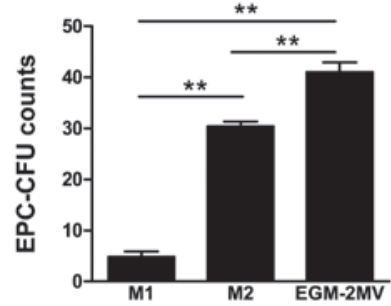

B

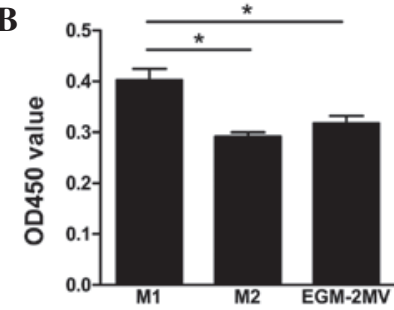

Figure 2. Endothelial progenitor cell (EPC) colony-forming capacity and proliferation in different culture media. (A) Mononuclear cells (MNCs) isolated form rat bone marrow were cultured in M1, M2 or EGM-2MV media, respectively. After 2 days, the non-adherent cells were collected and $1 \times 10^{6}$ cells were replated on a fibronectin-coated 24-well plate. On the 5th day, the number of colony-forming units (CFU) per well was counted. (B) A cell counting kit-8 assay was performed for late EPC proliferation. Data are presented as the mean $\pm \mathrm{SE}$ of four different experiments. ${ }^{*} \mathrm{P}<0.05$ and ${ }^{* *} \mathrm{P}<0.01$.

A

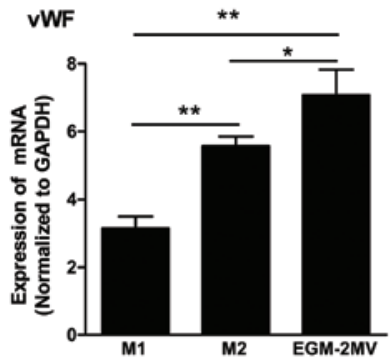

B

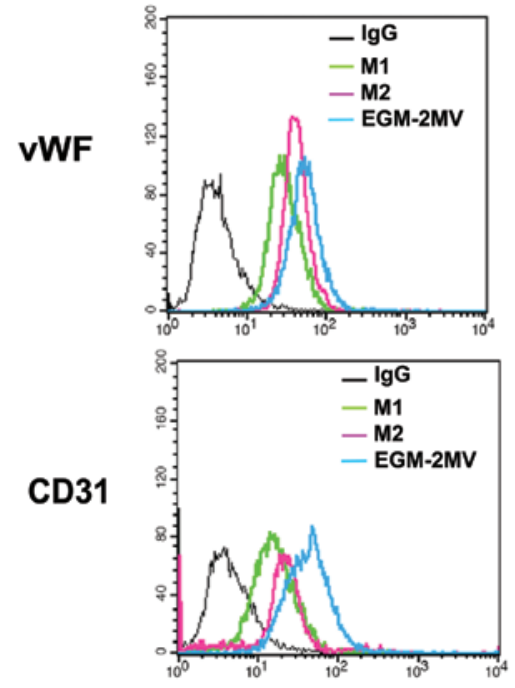

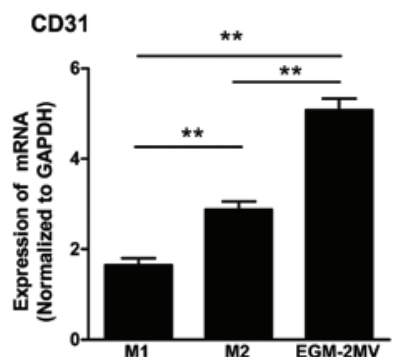
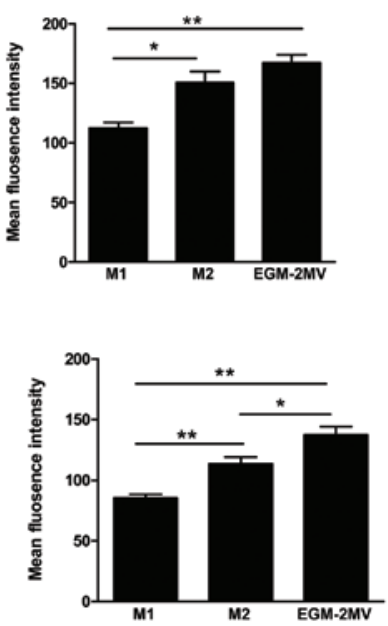

Figure 3. Late endothelial progenitor cell (EPC) differentiation in different culture media. Late EPCs were cultured in M1, M2 or EGM-2MV media, respectively. (A) The gene expression of von Willibrand Factor (vWF) and CD31 was then determined using qPCR. (B) Protein expression of vWF and CD31 was determined by a fluorescence-activated cell sorter (FACS). Representative FACS profiles of three independent experiments are shown in the left panels. Right panels show the relative fluorescence intensity that is normalized to the mean fluorescence intensity of isotype control. Data are presented as the mean \pm SE of four different experiments. ${ }^{*} \mathrm{P}<0.05$ and ${ }^{* *} \mathrm{P}<0.01$.

different media were round. After changing the medium on day four, attached cells were observed. Seven days following plating, the primary culture of MNCs started to differentiate into early EPCs (17) and tended to form colonies with the round cells at the center and the typical spindle cells at the periphery in M2 and EGM-2MV media, although the sizes of the colonies were different. The colonies grown in M2 media were smaller than those in EGM-2MV media. However, the attached cells in M1 media showed a fusiform shape. After 3 weeks, the 3rd or 4th generations of EPCs, namely late EPCs, with a cobblestone-like morphology similar to mature endothelial cells were grown to confluence in all media (Fig. 1).
EPC colony-forming capacity and proliferation in different culture media. Subsequent to seeding MNCs on wells, cells were incubated with the different culture media. Cells in EGM-2MV media exhibited the greatest colony forming capacity, followed by M2 and finally M1 media (Fig. 2A). The ability of late EPCs in M2 media to expand was low. However, late EPCs in M1 media reached confluence in a short period of time. Furthermore, the effects of different culture media on late EPC proliferation were analyzed by a CCK- 8 assay. In comparison to cells grown in M1 media, those in M2 and EGM-2MV media did not exhibit such a high level of cell proliferation (Fig. 2B). 
A

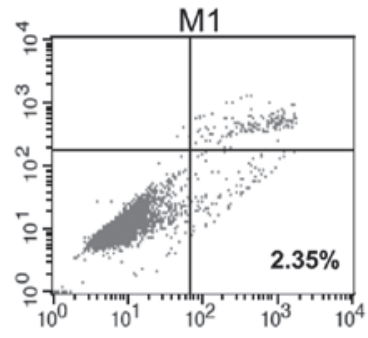

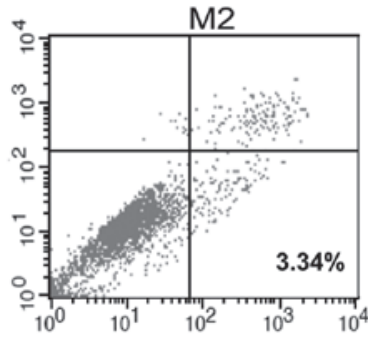

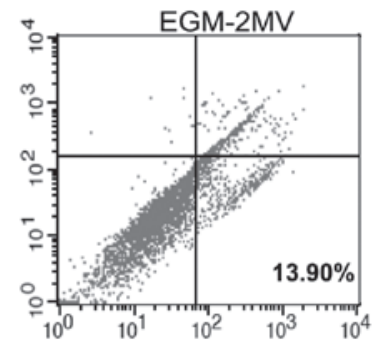

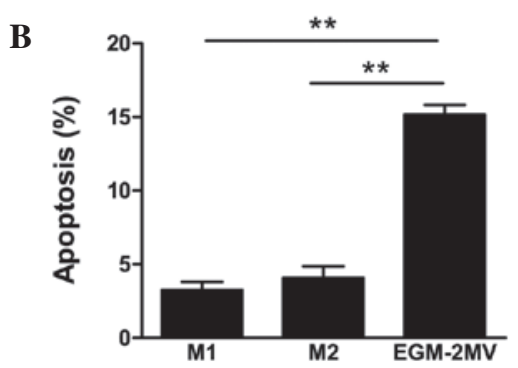

Figure 4. Endothelial progenitor cell (EPC) apoptosis in different culture media. (A) Late EPCs were cultured in M1, M2 or EGM-2MV media, respectively. Cells were harvested and the apoptotic cells were quantified by a fluorescence-activated cell sorter following Annexin $\mathrm{V}$ fluorescein isothiocyanate and propidium iodide staining. Annexin V-positive and PI-negative cells were defined as apoptotic. (B) The proportion of apoptotic cells. Data are presented as the mean $\pm \mathrm{SE}$ of four different experiments. ${ }^{* *} \mathrm{P}<0.01$.

Late EPC differentiation in different culture media. To identify the effects of different culture media on late EPC differentiation, the gene and protein expression of endothelial cell differentiation markers, such as vWF and CD31, was analyzed by qPCR and FACS. A number of cells were observed to express vWF and CD31 in each group, consistent with our previous results, which showed that cultured EPCs express endothelial cell differentiation markers (18). Moreover, late EPCs cultured in EGM-2MV media exhibited the highest gene and protein expression levels of CD31 and vWF (Fig. 3).

EPC apoptosis in different culture media. To determine the effects of different culture media on cell apoptosis, late EPCs were incubated in M1, M2 or EGM-2MV media. The cells were harvested and the apoptotic cells were quantified by FACS following Annexin V and PI staining. As shown in Fig. 4, late EPCs cultured with EGM-2MV medium exhibited significantly increased numbers of apoptotic cells, followed by M2 and finally M1 media.

EPC adhesion, migration and angiogenic properties in different culture media. As adhesion to the extracellular matrix is hypothesized to be important during novel blood vessel growth (19), the adhesion capacity of late EPCs cultured with different media was investigated. Quantitative analysis demonstrated that the number of cells that adhered to fibronectin was significantly higher in late EPCs cultured with EGM-2MV than those in the other groups (Fig. 5A).

The migratory function of EPCs in response to SDF-1 is also important during neovascularization and reendothelialization (19), and late EPCs have been shown to exhibit a greater migratory capacity than early EPCs in vitro (20). Thus, the effects of the different culture media on late EPC migration were analyzed by a modified Boyden chamber assay using SDF-1 as a chemoattractant. After 16 h, late EPCs cultured in
EGM-2MV exhibited the highest number of migrating cells among the three different groups (Fig. 5B).

It has been shown that late EPCs, but not early EPCs, successfully form capillary networks on Matrigel (6). An in vitro angiogenesis assay was performed with late EPCs to investigate the effects of the different culture media on EPC neovascularization. The functional capacity for tube formation of late EPCs on Matrigel was significantly stronger in the EGM-2MV group compared with the other groups (Fig. 5C).

NO production of late EPCs in different culture media. EPCs are able to secrete NO. NO is critical for regulating EPC functions (21). Therefore the effects of different culture media on the NO production in late EPCs were investigated. The culture media were collected, and the quantity of NO released from the late EPCs were determined. The results show that the level of NO was highest in the EGM-2MV group (Fig. 6).

\section{Discussion}

EPCs were initially isolated from adult human peripheral blood in 1997 by Asahara et al (22). Since the discovery, the original culture-based method to obtain human EPCs from blood has been adapted to mouse, pig and rat EPCs isolated from bone marrow $(12,14,17,23)$. MNCs isolated by density-gradient centrifugation from bone marrow were cultured with endothelial medium, which induced the MNCs to differentiate into EPCs.

Recent studies have suggested that the culture medium may be involved in the number and function of EPCs $(17,24)$. Therefore, in the present study, three common media were selected to culture EPCs isolated from rat bone marrow. The results have shown that EPCs exhibit different biological properties in different media.

In the conventional EPC culture, two predominant cell types have been shown to emerge from MNC cultures: Early and late EPCs (6). In the present study, after seven days, the primary 
A

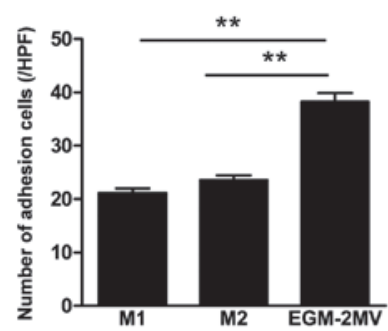

B

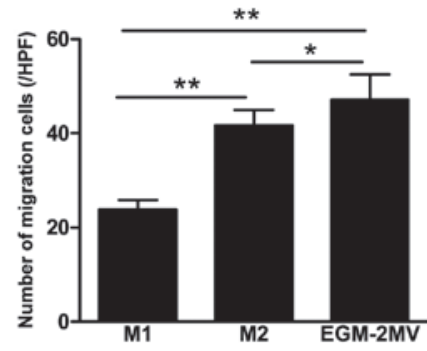

C
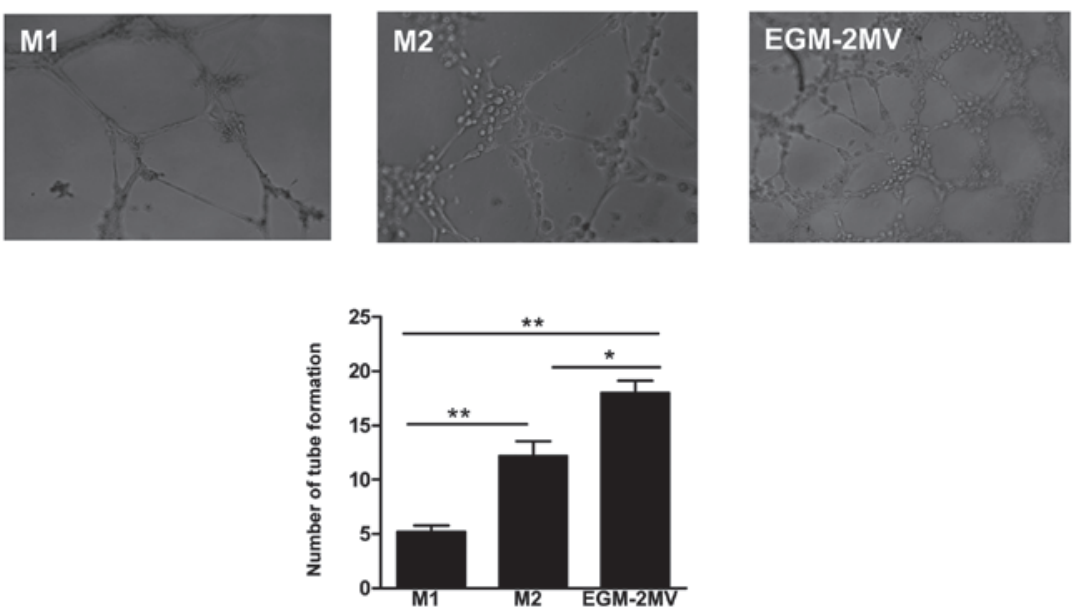

Figure 5. Endothelial progenitor cell (EPC) adhesion, migration and angiogenic properties in different culture media. Cultured late EPCs were reseeded on plate surfaces coated with fibronectin and incubated for $1 \mathrm{~h}$ at $37^{\circ} \mathrm{C}$. When non-adherent cells were removed by washing, adherent cells were fixed and stained with DAPI. DAPI positive cells were counted and averaged. (B) Late EPCs were cultured in M1, M2 or EGM-2MV media, respectively. Cells were harvested and $1 \times 10^{5}$ cells were placed in the upper chamber, and the lower chamber was filled with medium containg stromal cell-derived factor-1. After $16 \mathrm{~h}$, the migrated cells were stained with DAPI and analyzed. (C) Representative images of capillary networks formed by late EPCs cultured in different media (magnification, $x 100$ ). The complete tubes formed by cells were compared by computer software. Data are presented as the mean \pm SE of four different experiments. ${ }^{*} \mathrm{P}<0.05$ and ${ }^{* *} \mathrm{P}<0.01$.

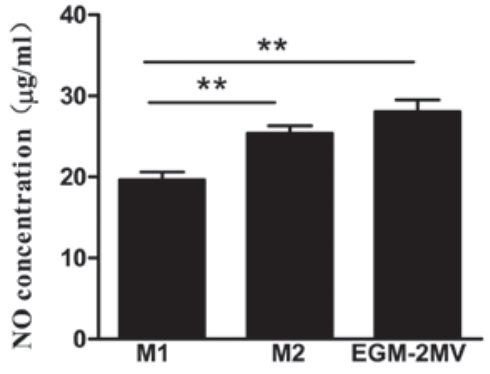

Figure 6. Nitric oxide (NO) production of late endothelial progenitor cells (EPCs) in different culture media. Late EPCs were cultured in M1, M2 or EGM-2MV media, respectively and the culture media were then collected. The quantity of $\mathrm{NO}$ was determined by the NO nitrate reductase assay. Data are presented as the mean $\pm \mathrm{SE}$ of four different experiments. ${ }^{* *} \mathrm{P}<0.01$.

culture of MNCs differentiating into early EPCs (17) showed different morphological phenotypes and exhibited different colony-forming capacities. The attached cells in M1 medium did not form the obvious clone and showed a fusiform shape. However, the cells cultured with M2 and EGM-2MV tended to form colonies with round cells at the center and the typical spindle cells at the periphery, with the colonies in M2 medium being smaller than those in EGM-2MV. A study by Yang et al (25) indicated that larger colonies exhibit greater differentiation than smaller colonies. In line with these results it was demonstrated that after three weeks, the 3rd or 4th generations of EPCs, namely late EPCs, cultured in EGM-2MV exhibited the highest gene and protein expression levels of CD31 and vWF, suggesting that endothelial differentiation may occur more robustly in cells cultured in EGM-2MV. In the present study it was also observed that late EPCs cultured in EGM-2MV exhibit a greater number of apoptotic cells. Ramasamy et al (26) demonstrated that elevated expression of stem cell-associated transcription factors is correlated with a reduction in cell apoptosis. As EGM-2MV is the medium that most strongly supports the differentiation of EPCs towards endothelial cells, it is reasonable that a greater number of apoptotic cells were observed in late EPCs in the EGM-2MV medium.

Cell proliferation is markedly affected by culture conditions, particularly the addition of cytokines. When the required cytokines were not added to the culture media, cell profanation was slower and cell death occurred. In the present study it was demonstrated that early EPCs cultured in EGM-2MV exhibited the highest colony forming capacity, followed by those cultured in M2 and finally in M1 media. However, M1 greatest effect on the promotion of proliferation of late EPCs. These results indicated that the requirement of cytokines is different during EPC maturation. Jianguo et al (17) demonstrated that combinations of cytokines increased the rate of proliferation of EPCs. It is therefore reasonable that M1 was identified to exhibit the greatest effect on late-EPC proliferation as the bovine pituitary extract contained more than one type of cytokine.

The Matrigel model is a global assay, which analyzes multiple cellular processes involved in blood vessel growth, such as cell migration, adhesion and differentiation. In the present 
study, it was demonstrated that the number cell that adhered to fibronectin and migrated in response to SDF-1 was significantly higher in late EPCs cultured with EGM-2MV. Thus, EGM-2MV medium was also identified to markedly increase the number of cell extensions formed within cell networks.

In the present study, it was also observed that the production of NO in late EPCs was greatest when cultured in EGM-2MV medium. NO is a predominant vasodilator and a key survival factor for the endothelium. Endothelial dysfunction is characterized by low bioavailability of endothelium-derived NO, which itself is an independent predictor of future cardiovascular events (27). Ozuyaman et al (28) demonstrated that NO stimulates EPC mobilization from bone marrow stem cell niches to the peripheral circulation, implying that they participate in the neovascularization process (28). Moreover, $\mathrm{NO}$ is essential for the survival, migration and angiogenesis of EPCs (29). This indicates the importance of NO in maintaining EPC function. These results suggest the possibility that the increased NO production in EGM-2MV cultured cells contributes to the promotion of EPC functions, such as migration, adhesion and tube formation in vitro. The biological properties of bone marrow-derived early and late EPCs were observed in vitro. However, further investigation is required to determine the functions of EPCs cultured in the different media in vivo.

In conclusion, ex vivo culture and expansion of EPCs may be a promising strategy to overcome the clinical problem of limited cell numbers; however, cell culture condition, for example cell media, affect the biological properties of bone marrow-derived early and late EPCs.

\section{Acknowledgements}

This study was supported by the National Natural Science Foundation of China (grant nos. 30900290 and 31270993); the Natural Science Foundation of Shandong Province (grant nos. ZR2009CQ027 and ZR2010HQ046); the Program for New Century Excellent Talents in University (grant no. NCET-100922); the Foundation of Shandong Educational Committee (grant no. J09LF06); and the Project-sponsored by SRF for ROCS, SEM. The authors would like to thank Dr Emil Avsar for his critical reading of the manuscript.

\section{References}

1. Vita JA: Endothelial function. Circulation 124: e906-e912, 2011.

2. Möbius-Winkler S, Höllriegel R, Schuler G and Adams V: Endothelial progenitor cells: implications for cardiovascular disease. Cytometry A 75: 25-37, 2009.

3. Ben-Shoshan J and George J: Endothelial progenitor cells as therapeutic vectors in cardiovascular disorders: from experimental models to human trials. Pharmacol Ther 115: 25-36, 2007

4. Urbich C, Aicher A, Heeschen C, et al: Soluble factors released by endothelial progenitor cells promote migration of endothelial cells and cardiac resident progenitor cells. J Mol Cell Cardiol 39: 733-742, 2005

5. Wang TJ, Yang YJ, Xu B, et al: Atorvastatin accelerates both neointimal coverage and re-endothelialization after sirolimus-eluting stent implantation in a porcine model: new findings from optical coherence tomography and pathology. Circ J 76: 2561-2571, 2012

6. Hur J, Yoon CH, Kim HS, et al: Characterization of two types of endothelial progenitor cells and their different contributions to neovasculogenesis. Arterioscler Thromb Vasc Biol 24: 288-293, 2004.
7. Brown MA, Wallace CS, Angelos $M$ and Truskey GA: Characterization of umbilical cord blood-derived late outgrowth endothelial progenitor cells exposed to laminar shear stress. Tissue Eng Part A 15: 3575-3587, 2009.

8. Aburakawa Y, Kawabe J, Okada M, et al: Prostacyclin stimulated integrin-dependent angiogenic effects of endothelial progenitor cells and mediated potent circulation recovery in ischemic hind limb model. Circ J 77: 1053-1062, 2013.

9. Iwaguro H, Yamaguchi J, Kalka C, et al: Endothelial progenitor cell vascular endothelial growth factor gene transfer for vascular regeneration. Circulation 105: 732-738, 2002.

10. Taniguchi E, Kin M, Torimura T, et al: Endothelial progenitor cell transplantation improves the survival following liver injury in mice. Gastroenterology 130: 521-531, 2006.

11. Loomans CJ, de Koning EJ, Staal FJ, et al: Endothelial progenitor cell dysfunction: a novel concept in the pathogenesis of vascular complications of type 1 diabetes. Diabetes 53: 195-199, 2004.

12. Yang N, Li D, Jiao P, et al: The characteristics of endothelial progenitor cells derived from mononuclear cells of rat bone marrow in different culture conditions. Cytotechnology 63: 217-226, 2011.

13. Ye C, Bai L, Yan ZQ, Wang YH and Jiang ZL: Shear stress and vascular smooth muscle cells promote endothelial differentiation of endothelial progenitor cells via activation of Akt. Clin Biomech (Bristol, Avon) 23 (Suppl 1): S118-S124, 2008.

14. Zhang X, Cui X, Cheng L, et al: Actin stabilization by jasplakinolide affects the function of bone marrow-derived late endothelial progenitor cells. PLoS One 7: e50899, 2012.

15. Tepper OM, Galiano RD, Capla JM, et al: Human endothelial progenitor cells from type II diabetics exhibit impaired proliferation, adhesion, and incorporation into vascular structures. Circulation 106: 2781-2786, 2002.

16. Chen YH, Lin SJ, Lin FY, et al: High glucose impairs early and late endothelial progenitor cells by modifying nitric oxide-related but not oxidative stress-mediated mechanisms. Diabetes 56: 1559-1568, 2007

17. Jianguo W, Tianhang L, Hong Z, et al: Optimization of culture conditions for endothelial progenitor cells from porcine bone marrow in vitro. Cell Prolif 43: 418-426, 2010.

18. Cui X, Zhang X, Guan X, et al: Shear stress augments the endothelial cell differentiation marker expression in late EPCs by upregulating integrins. Biochem Biophys Res Commun 425: 419-425, 2012.

19. Urbich $C$ and Dimmeler S: Endothelial progenitor cells: characterization and role in vascular biology. Circ Res 95: 343-353, 2004.

20. Yoon CH, Hur J, Park KW, et al: Synergistic neovascularization by mixed transplantation of early endothelial progenitor cells and late outgrowth endothelial cells: the role of angiogenic cytokines and matrix metalloproteinases. Circulation 112: 1618-1627, 2005.

21. Hamed S, Brenner B and Roguin A: Nitric oxide: a key factor behind the dysfunctionality of endothelial progenitor cells in diabetes mellitus type-2. Cardiovasc Res 91: 9-15, 2011.

22. Asahara T, Murohara T, Sullivan A, et al: Isolation of putative progenitor endothelial cells for angiogenesis. Science 275: 964-967, 1997.

23. Wang QR, Wang BH, Huang YH, Dai G, Li WM and Yan Q: Purification and growth of endothelial progenitor cells from murine bone marrow mononuclear cells. J Cell Biochem 103: 21-29, 2008.

24. Muscari C, Gamberini C, Basile I, et al: Comparison between culture conditions improving growth and differentiation of blood and bone marrow cells committed to the endothelial cell lineage. Biol Proced Online 12: 9023, 2010.

25. Yang J, Ii M, Kamei N, et al: $\mathrm{CD} 34^{+}$cells represent highly functional endothelial progenitor cells in murine bone marrow. PLoS One 6: e20219, 2011.

26. Ramasamy R, Tong CK, Yip WK, Vellasamy S, Tan BC and Seow HF: Basic fibroblast growth factor modulates cell cycle of human umbilical cord-derived mesenchymal stem cells. Cell Prolif 45: 132-139, 2012.

27. Desjardins F and Balligand JL: Nitric oxide-dependent endothelial function and cardiovascular disease. Acta Clin Belg 61: 326-334, 2006

28. Ozüyaman B, Ebner P, Niesler U, et al: Nitric oxide differentially regulates proliferation and mobilization of endothelial progenitor cells but not of hematopoietic stem cells. Thromb Haemost 94: 770-772, 2005

29. He T, Peterson TE, Holmuhamedov EL, et al: Human endothelial progenitor cells tolerate oxidative stress due to intrinsically high expression of manganese superoxide dismutase. Arterioscler Thromb Vasc Biol 24: 2021-2027, 2004. 